\title{
PAEDIATRIC STROKE- AN ANALYSIS ON ARTERY OF PAEDIATRIC INFARCT- LENTICULOSTRIATE ARTERIES
}

\author{
Sukumar Ramasamy1, Periakaruppan Alagappan², Viswanathan Venkataraman³, Amarnath Chellathurai ${ }^{4}$, Suvec Alagu Sethuraman \\ Alagarsamy5, Praveen Kumar Mani ${ }^{6}$
}

1 Professor, Government Stanley Medical College, Chennai, Tamilnadu. 2Professor, Government Stanley Medical College, Chennai, Tamilnadu. 3 Professor, Kanchi Kamakoti Childs Trust Hospital, Chennai, Tamilnadu. ${ }^{4}$ Professor, Government Stanley Medical College, Chennai, Tamilnadu. ${ }^{5}$ Resident, Government Stanley Medical College, Chennai, Tamilnadu. ${ }^{6}$ Resident, Government Stanley Medical College, Chennai, Tamilnadu.

\section{BACKGROUND}

ABSTRACT

Stroke is a neurological insult due to cut-off of blood supply to brain.[1] Paediatric stroke is a rare, but increasing cause of childhood morbidity.[2,3] Causes and pattern of stroke in children and newborn is different from adults. Stroke can be haemorrhagic or ischaemic or both. In India, paediatric population usually refers to children under 12 years.

The purpose is to study the vascular distribution pattern and prevalence of isolated involvement of lenticulostriate arteries in acute arterial stroke among paediatric population in India using MRI.

\section{MATERIALS AND METHODS}

This is a cross-sectional study involving 144 paediatric patients from newborn to 12 years of age in the tertiary care medical college hospital, India. Study included patients diagnosed to have acute arterial infarct by MRI study of brain. The areas and arterial territory of the infarct were studied and analysed. Isolated involvement of medial and lateral lenticulostriate arteries were analysed. Statistical analysis was done by Chi-Square test and Fisher's exact test.

\section{RESULTS}

Our study shows isolated involvement of Lenticulostriate arteries accounting for $51.4 \%$ of acute arterial infarct in paediatric population. There is increased incidence of stroke in Lenticulostriate arterial territory in 0 - 4 years of age group (P value- 0.016 ). Lateral lenticulostriate arteries were commonly involved than medial, which accounts for $85 \%$ of their total.

\section{CONCLUSION}

Involvement of vascular territory in paediatric age group is different from adults. Among paediatric arterial infarcts, Lenticulostriate arteries are commonly affected, lateral more than medial. There is increased incidence of Lenticulostriate artery infarct in $0-4$ yrs. of age. Since infarct in Lenticulostriate artery accounts for more than half of acute arterial infarct in paediatric population, it can be given a special name as "Artery of Paediatric Infarct."

\section{KEY WORDS}

Paediatric Stroke, Lenticulostriate Artery, Lenticulostriate Vasculopathy, Arterial Infarct.

HOW TO CITE THIS ARTICLE: Ramasamy S, Alagappan P, Venkataraman V, et al. Paediatric stroke- an analysis on artery of paediatric infarct- lenticulostriate arteries. J. Evolution Med. Dent. Sci. 2018;7(21):2550-2554, DOI: 10.14260/jemds/2018/574

\section{BACKGROUND}

Stroke is a neurological insult due to cut-off of blood supply to brain. ${ }^{[1]}$ Paediatric stroke is a rare, but increasing cause of childhood morbidity.[2,3] Causes and pattern of stroke in children and newborn is different from adults. Stroke can be haemorrhagic or ischaemic or both. In India, paediatric population usually refers to children under 12 years. ${ }^{[4]}$ Diagnosis and reporting of paediatric stroke has been increasing in last two decades due to increase in health care facility in India. MRI very much helps in diagnosis and evaluation of stroke. Strokes can occur in the prenatal or

'Financial or Other Competing Interest': None.

Submission 22-03-2018, Peer Review 06-05-2018,

Acceptance 12-05-2018, Published 21-05-2018.

Corresponding Author:

Dr. Periakaruppan Alagappan,

301, Department of Radio-Diagnosis,

Government Stanley Medical College,

Chennai-600001, Tamilnadu.

E-mail:amarrd02@yahoo.co.in

DOI: $10.14260 /$ jemds $/ 2018 / 574$ postnatal period. Causes of prenatal stroke can be due to congenital heart disease, coagulation disorder, infections and other causes. Postnatal causes of stroke include congenital heart disease, head trauma, diseases affecting intracranial arteries, infections and other causes. Symptoms of antenatal stroke may go unrecognised in the postnatal period or may present as seizure, lethargy, irritability, focal neurological deficit in early childhood. In older children it presents as weakness, headache, vomiting, blurring of vision, new-onset seizure and dizziness.[1,5] Stroke can be due to arterial ischaemia, venous thrombosis or haemorrhage. Arterial ischaemic stroke accounts for more than $80 \%$ of total paediatric stroke.[2] Purpose of the study is to analyse vascular territory distribution of acute arterial infarct in paediatric patients less than 12 years of age and assess involvement of Lenticulostriate artery (LSA).

\section{MATERIALS AND METHODS}

Children less than 12 years of age referred with symptoms and findings of acute stroke for MRI were analysed and who had arterial infarct were taken into study. Symptoms of 
stroke includes focal neurological deficit, focal seizure, lethargy, irritability, headache, vomiting and blurring of vision. Patients with acute infarct have infarct area, which appears hyperintense on diffusion weighted images and hypointense on Apparent Diffusion Coefficient (ADC) Maps.[6] Signs of arterial infarct includes distribution in arterial territory and cut-off or narrowing in supplying artery in MR Angiogram. MR angiogram may not show features of occlusion in small lacunar infarcts. CT brain was also done in 26 patients before doing MRI, 6 of them revealed tiny specks of calcification in basal ganglia which is supplied by lenticulostriate arteries (Figure 1).

Study was cross-sectional study done at our institution from December 2011 to December 2016. Ethical committee clearance was obtained for the study. Parental consent was obtained for each case. MRI brain was done for patients with symptoms suggestive of stroke and patients who had acute arterial infarct were taken into study. Exclusion criteria were other causes of infarct and infarct mimics including venous infarct, congenital heart disease, meningitis, vasculitis, granulomas, acute demyelination, post-traumatic, postsurgical and brain tumour cases. MR findings of the patients were analysed and studied.

The area of infarct followed either arterial or venous territory or no territory as in cases of infection. Major intracranial arteries include Middle Cerebral Artery (MCA), Anterior Cerebral Artery (ACA), Posterior Cerebral Artery (PCA), vertebral and basilar arteries. The infarcted territory varied depending on the causes. Among the smaller arteries, Lenticulostriate arteries are our arteries of interest. Medial lenticulostriate artery originates from proximal part of anterior cerebral artery (A1 Segment) and supplies most of globus pallidus, anterior aspect of caudate, the anterior limb and genu of the internal capsule. Lateral striate arteries arise from proximal segment of Middle cerebral artery (M1) and supplies most of Putamen, lateral part of globus pallidus, part of caudate, external capsule and upper internal capsule.[7-9] Laboratory assessment was done which included specific laboratory tests to look for specific causes like coagulopathies, titre for varicella zoster, haematological disorders or CNS infections. Complete blood count with platelet count, Blood coagulation profile, Serum and CSF routine + lactate/ pyruvate, haemoglobin electrophoresis, Liver and Renal Function tests, Echo and ECG.

\section{MRI Protocol}

MRI brain was done using Siemens Magnetom AERA 48 Channel 1.5 Tesla Machine. MRI was done using standardised protocol for stroke (T2W, T1W, SWI, DWI, ADC MAPPING, T2W FLAIR, MR Venogram and 3D TOF Angiogram). The studies were reported by radiologists with more than 10 years of experience.

\section{Statistical Analysis}

To compare proportions between groups, Chi-square test is applied. If any expected cell frequency is less than five, then Fisher's exact test is used. To analyse the data SPSS (IBM SPSS Statistics for Windows, Version 22.0, Armonk, NY: IBM Corp. Released 2013) is used. Significance level is fixed as $5 \%$ $(\alpha=0.05)$.

\section{RESULTS}

Patients with less than 12 years of age who had infarct were 277 in number, among them 211 had acute infarct which showed areas of diffusion restriction and 66 had chronic infarcts. Out of 211 patients with acute infarct 144 patients had infarct due to arterial ischaemia, 17 due to germinal matrix haemorrhages, 13 due to venous infarct, 10 patients due to tuberculous meningitis, 8 due to moyamoya disease, 6 due to vasculitis, 5 due to mitochondrial diseases, 4 due to congenital heart disease due to embolic stroke, 3 due to herpes encephalitis and 1 due to sickle cell disease. We analysed our study population of 144 patients who had acute arterial infarct.

Among the 144 patients with acute arterial infarct, 63 had involvement of one major artery and its territory. Among them 49 involving MCA, 10 posterior cerebral artery and 4 due to anterior cerebral artery involvement (Table I). One or more major arteries and its territories was involved in 7 cases. 74 patients had isolated involvement of Lenticulostriate arteries. Isolated involvement of lateral lenticulostriate artery territory showing diffusion restriction in MRI accounted for 63 cases (Figure 2) and medial lenticulostriate artery territory in 11 cases (Figure 3).

Infarcts in lenticulostriate artery territory accounts for $51.4 \%$, MCA $34 \%$, PCA $6.9 \%$, ACA $2 \%$ and multiple territories are involved in $4.8 \%$. Infarct in LSA territory is common in 0 4 years' age than in $4-12$ years' age group ( $\mathrm{p}$-value 0.016 ) There is no sex predilection in LSA infarcts ( $p$-value 0.935 ). There is also no left or right-sided lateralisation ( $p$-value 0.349). Similarly, there is no lateralisation or sex predilection among medial and lateral LSA territories (Table II). There is increased incidence of infarct in lateral LSA compared to medial LSA, which accounts for 85\% of LSA infarction.

Median age of LSA infarct is less (3 years) compared to MCA territory infarct (6 years). There is predominance of acute arterial infarct in lenticulostriate territory in less than 4 yrs. of age (Graph 1) and it accounts for $51.4 \%$ of total acute arterial infarct under 12 years of age in our study and $66.6 \%$ in 0 to 4 years of age (Table III).

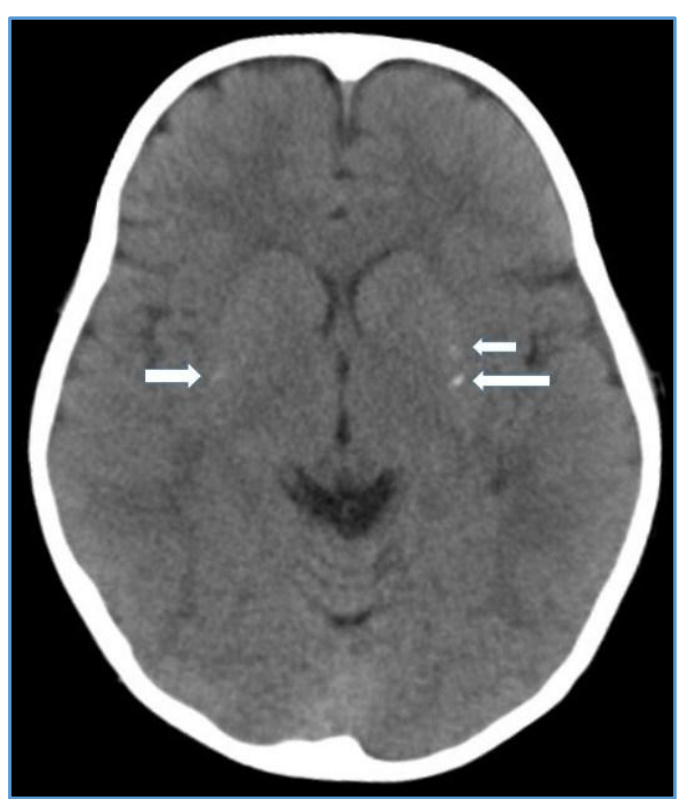

Figure 1. NECT Brain of 1-year-old Male child, showing specks of Calcification in Bilateral Lenticulostriate Artery Territory (arrows pointing the Hyperdense Calcific Foci) 


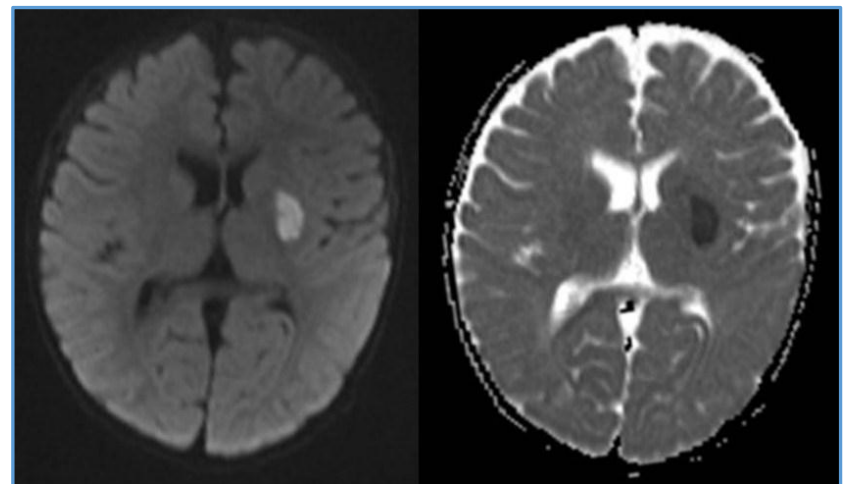

Figure 2. MRI Brain of a 2-year-old Male child. Diffusion Weighted Image (b-1000) and its corresponding ADC image showing Acute Infarct in Lateral Lenticulostriate Artery Territory

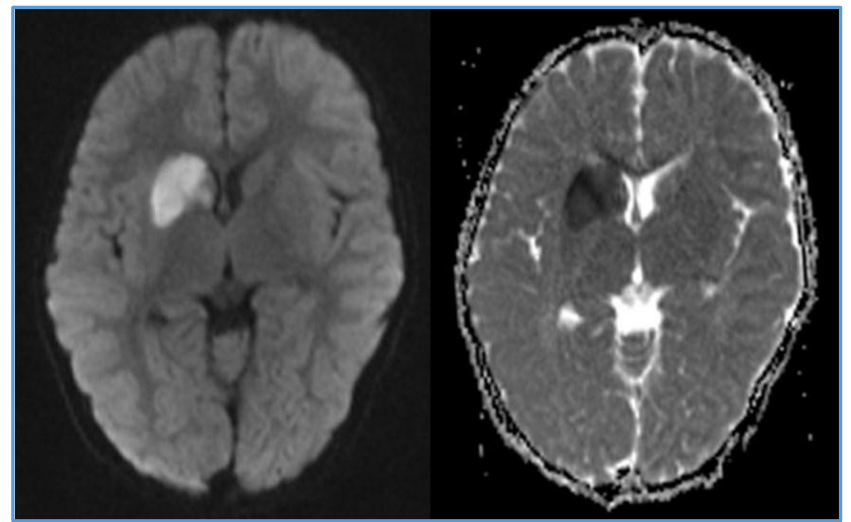

Figure 3. MRI Brain of a 5-year-old Male child. Diffusion Weighted Image (b-1000) and its corresponding ADC image showing Acute Infarct in Medial Lenticulostriate Artery Territory

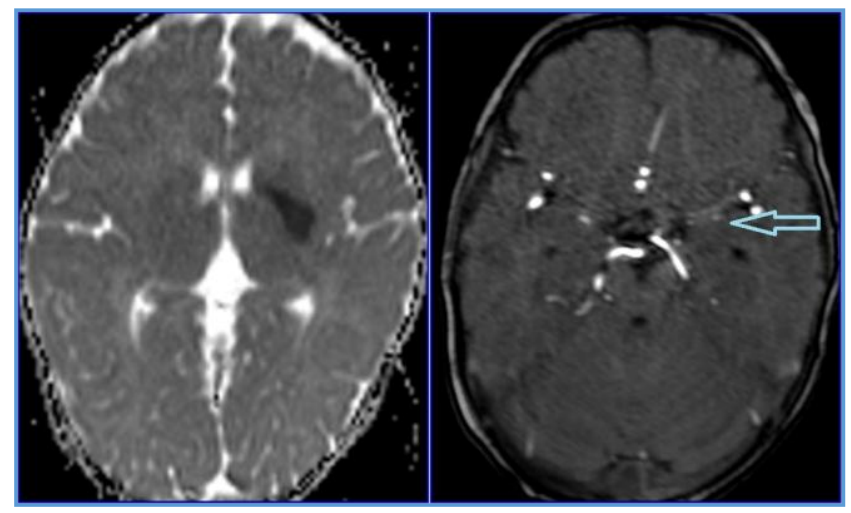

Figure 4. MRI Brain of a 3-year-old Female child. ADC image showing Acute Infarct in Medial Lenticulostriate Artery Territory. MRA shows narrowed Medial Lenticulostriate Artery

\begin{tabular}{|c|c|c|c|c|c|}
\hline Age Group & $\begin{array}{c}\text { Total Acute } \\
\text { Arterial } \\
\text { Infarct }\end{array}$ & LSA & MCA & PCA & ACA \\
\hline $0-1$ & 19 & 9 & 5 & 2 & 1 \\
\hline $1-2$ & 31 & 23 & 7 & 1 & 0 \\
\hline $2-3$ & 15 & 11 & 4 & 0 & 0 \\
\hline $3-4$ & 16 & 11 & 4 & 1 & 0 \\
\hline $4-5$ & 6 & 4 & 2 & 0 & 0 \\
\hline $5-6$ & 9 & 3 & 4 & 1 & 0 \\
\hline $6-7$ & 7 & 3 & 3 & 0 & 1 \\
\hline
\end{tabular}

\begin{tabular}{|c|c|c|c|c|c|}
\hline $7-8$ & 5 & 1 & 2 & 1 & 0 \\
\hline $8-9$ & 11 & 4 & 5 & 1 & 1 \\
\hline $9-10$ & 5 & 1 & 3 & 1 & 0 \\
\hline $10-11$ & 9 & 3 & 5 & 1 & 0 \\
\hline $11-12$ & 11 & 1 & 5 & 1 & 1 \\
\hline Total & 144 & $\mathbf{7 4}$ & $\mathbf{4 9}$ & $\mathbf{1 0}$ & $\mathbf{4}$ \\
\hline Table I. Distribution of Acute Infarct in each Arterial \\
Territory \\
\hline
\end{tabular}

${ }^{*}$ One or more territories is involved in 7 cases.

\begin{tabular}{|c|c|c|c|}
\hline Age & Medial LSA & Lateral LSA & Total \\
\hline $0-1$ & 0 & 9 & 9 \\
\hline $1-2$ & 4 & 19 & 23 \\
\hline $2-3$ & 2 & 9 & 11 \\
\hline $3-4$ & 1 & 10 & 11 \\
\hline $4-5$ & 1 & 3 & 4 \\
\hline $5-6$ & 0 & 3 & 3 \\
\hline $6-7$ & 1 & 2 & 3 \\
\hline $7-8$ & 0 & 1 & 1 \\
\hline $8-9$ & 1 & 3 & 4 \\
\hline $9-10$ & 0 & 1 & 1 \\
\hline $10-11$ & 1 & 2 & 3 \\
\hline $11-12$ & 0 & 1 & 1 \\
\hline Total & $\mathbf{1 1}$ & $\mathbf{6 3}$ & $\mathbf{7 4}$ \\
\hline
\end{tabular}

Table II. Distribution of Infarct in Medial and Lateral LSA by Age Group

\begin{tabular}{|c|c|c|c|c|}
\hline $\begin{array}{l}\text { Age } \\
\text { Group }\end{array}$ & \begin{tabular}{|c|} 
Total \\
Number of \\
Acute \\
Arterial \\
Infarcts \\
\end{tabular} & $\begin{array}{c}\text { Number of } \\
\text { Infarcts in } \\
\text { LSA } \\
\text { Territory }\end{array}$ & $\%$ & $\begin{array}{c}\text { Chi- } \\
\text { Square } \\
\text { Test }\end{array}$ \\
\hline $0-4$ years & 81 & 54 & $66.6 \%$ & \multirow{3}{*}{$\begin{array}{c}\text { P-Value } \\
0.016\end{array}$} \\
\hline $\begin{array}{c}4-12 \\
\text { years }\end{array}$ & 63 & 20 & $31.7 \%$ & \\
\hline Total & 144 & 74 & $51.3 \%$ & \\
\hline $\begin{array}{c}\text { Table III } \\
\text { and }\end{array}$ & $\begin{array}{l}\text { ompariso } \\
\text { arct in } L\end{array}$ & Infar & $\begin{array}{l}\text { cute Art } \\
-4 \text { vs. } 4 \\
\end{array}$ & $\begin{array}{l}\text { al Infarct } \\
2 \text { yrs. }\end{array}$ \\
\hline
\end{tabular}

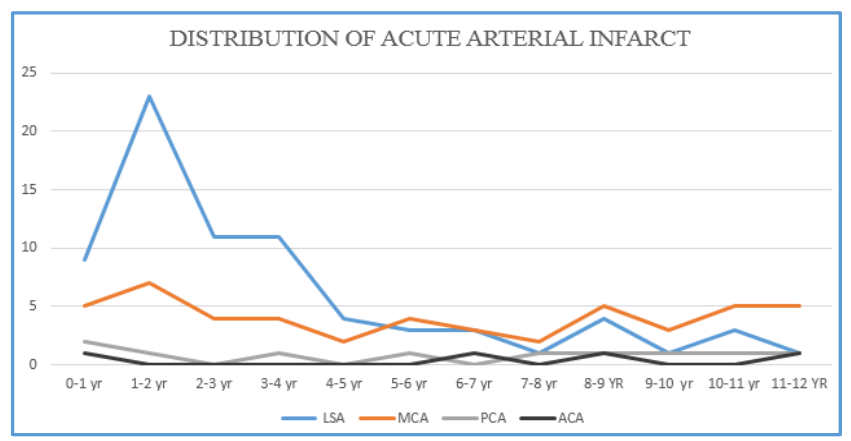

Graph 1. Distribution of Acute Arterial Infarcts by Age Group

\section{DISCUSSION}

Incidence of paediatric stroke has been increasingly recognised in last few decades in India.[1] Only few case studies are available in India on paediatric stroke.[1],[2] Various causes has been attributed for stroke in paediatric population. Initially, the term mineralising vasculopathy was used in late 1960s to describe calcification in basal ganglia on CT brain.[10-13] Few of our cases showed calcification in basal ganglia in NECT brain.

Lenticulostriate vasculopathy (LSV) was introduced by Grant et al in 1985 as mineralisation was not present in all 
patients with changes in basal ganglia and thalami.[10-12] Initially, the term Lenticulostriate vasculopathy was used to refer changes in lenticulostriate artery territory in transcranial ultrasound in neonates and infants and later studies included age upto 18 months of age. Initially, it was thought that it is an uncommon entity. Thereafter, more than 30 studies on lenticulostriate vasculopathy have been published. Various causes have been proposed to lenticulostriate vasculopathy like perinatal asphyxia, respiratory disease, congenital heart disease, foetal TORCH infection, chromosomal aberrations, congenital malformations, neonatal polycythaemia, neonatal hypoglycaemia, bacterial meningitis, congenital metabolic acidosis, sialidosis, foetal hydrops, neonatal lupus erythematosus and idiopathic.[10-13] LSV does not refer to arterial infarct occurring in its territory, rather any changes in its territory in Ultrasound, CT or MRI Brain. The causal relation between LSV and acute infarcts are not proved by studies. Few case reports have shown ischaemic infarct occurring in cases of LSV.[14] Earlier studies have shown increased involvement of lenticulostriate arteries in various diseases of paediatric population other than stroke. LSV is a term which is used mainly to describe sonographic findings of results upto 2 years of age.[10,11,13] As transcranial ultrasound cannot be performed after fusion of fontanelles, it cannot be assessed after 2 years of age. Hence, causal relationship between infarct and LSV cannot be established in all cases.

Our study on acute arterial infarct in paediatric population shows higher incidence of arterial infarcts occurring in lenticulostriate territory with no specific risk factor common among them. No previous studies have been done to assess territorial distribution of infarcts in paediatric population. Many of the patients in our study had history of trivial trauma just before the episode, as it was observed in earlier studies.[15-17] Various causes have been attributed in literature for increased involvement of lenticulostriate arteries like rigid mineralised artery increases vulnerability of vessels to shear injury during trauma and acute angle of origin of lenticulostriate arteries in paediatric age group increasing the shear force even during mild trauma.[15-18]

Transient cerebral arteriopathy (TCA) is a unilateral monophasic arteriopathy frequently recognised in children with arterial ischaemic stroke usually affecting proximal middle or anterior cerebral artery and its branches.[19-21] Diagnosis of TCA requires that follow-up imaging at 6 months post-stroke shows neither additional stenoses nor progression of the original stenosis. Various causes has been attributed for the development of TCA which mainly include infections like varicella zoster, enteroviruses, parvovirus B19, Mycoplasma pneumonia and Borrelia burgdorferi.[21] Later the term Focal Cerebral Arteriopathy (FCA) was introduced by International Paediatric Stroke Study (IPSS) as an entity which has stenosis on vascular imaging, not otherwise classified as dissection, moyamoya, sickle cell arteriopathy, post-varicella arteriopathy, vasculitis or other specific diagnoses (such as post-irradiation arteriopathy) and thus includes TCA in its definition.[19-21] Studies have shown some viral infections as predisposing factor for TCA.[19-22]

Lenticulostriate vasculopathy and TCA are two different terms, which describe changes or pathology in lenticulostriate arterial territory, the former refers to younger age group upto 2 years of age and later upto 18 years of age. However, their causal relationship between LSV progressing to TCA is not yet established.

Limitations in our study includes inclusion of cases of only acute arterial stroke, follow-up imaging was not done for all cases, DSA cerebral angiogram was not done to visualise tiny branches of lenticulostriate arteries and transcranial ultrasonography was not done in neonatal cases.

In our study of 144 patients with acute arterial infarct, there is increased incidence of the infarct in LSA territory accounting for $51.4 \%$ of arterial stroke in paediatric age group and infarcts occur more in lateral lenticulostriate artery territory. Median age of infarct is less in LSA territory than MCA territory. There is no lateralisation or sex predilection in infarcts in LSA territory.

According to various studies, the incidence of acute arterial infarct among Indian adults in MCA, PCA, ACA and multiple territories are 50\% - 80\%, 10\% - 15\%, 5\% - 10\% and 5\% - 6\% respectively.[23-25] MCA territory infarcts account for $50 \%-80 \%$ of total arterial stroke among adults in India, while in our study paediatric arterial infarct predominantly involved isolated LSA territory accounting for $51.4 \%$ of total and $66.6 \%$ in $0-4$ years of age. No studies on incidence of LSA in adults in India is available. In our study, isolated LSA infarcts are common than MCA territory infarcts in paediatric population and shows significantly increased incidence in 0 - 4 years of age.

\section{CONCLUSION}

Involvement of vascular territory in paediatric age group is different from adults. Among paediatric arterial infarcts Lenticulostriate arteries are commonly affected, lateral more than medial. There is increased incidence of Lenticulostriate artery infarct in $0-4$ years of age. Since infarct in Lenticulostriate artery accounts for more than half of acute arterial infarct in paediatric population, it can be given a special name as "Artery of Paediatric Infarct."

\section{What is Already Known?}

Arterial ischaemic stroke accounts for $80 \%-90 \%$ of infarct in paediatric population in India.

\section{What this Study Adds?}

Acute arterial stroke occurs most commonly in Lenticulostriate arterial territory in paediatric population, lateral more than medial and there is significantly increased incidence of infarct in LSA under 4 years of age.

\section{REFERENCES}

[1] Patra C, Sarkar S, Guha D, et al. Clinico-etiological profile of childhood stroke in a tertiary care hospital in Eastern India. Journal of Neurosciences in Rural Practice 2015;6(4):515-9.

[2] Parakh M, Arora V, Khilery B. A prospective study evaluating the clinical profile of pediatric stroke in Western Rajasthan. Journal of Neurological Disorders 2014;2(6):1-4.

[3] Indian pediatrics. Cereberovascular disease in children.

http://www.indianpediatrics.net/feb2000/personal.h tm. Accessed April 20, 2017. 
[4] Indian pediatrics. IAP Policy on age of children for Pediatric care. http://www.indianpediatrics.net/may1999/may-461463.htm. Accessed April 20, 2017.

[5] Tsze DS, Valente JH. Pediatric stroke: a review. Emergency Medicine International 2011;2011:734506.

[6] Allen LM, Hasso AN, Handwerker J, et al. Sequencespecific MR imaging findings that are useful in dating ischemic stroke. Radiographics 2012;32(5):1285-97.

[7] Kang HS, Han MH, Kwon BJ, et al. Evaluation of the lenticulostriate arteries with rotational angiography and 3D reconstruction. American Journal of Neuroradiology 2005;26(2):306-12.

[8] Berman SA, Hayman LA, Hinck VC. Correlation of CT cerebral vascular territories with function: 3. Middle cerebral artery. American Journal of Roentgenology 1984;142(5):1035-40.

[9] Berman SA, Hayman LA, Hinck VC. Correlation of CT cerebral vascular territories with function: I. Anterior cerebral artery. American Journal of Roentgenology 1980;135(2):253-7.

[10] Wang HS, Kuo MF, Chang TC. Sonographic lenticulostriate vasculopathy in infants: some associations and a hypothesis. American Journal of Neuroradiology 1995;16(1):97-102.

[11] Sisman J, Logan JW, Westra SJ, et al. Lenticulostriate vasculopathy in extremely low gestational age newborns: Inter-rated variability of cranial ultrasound readings, antecedents and postnatal characteristics. Journal of Pediatric Neurology 2014;12(4):183-93.

[12] Grant EG, Williams AL, Schellinger D, et al. Intracranial calcification in the infant and neonate: evaluation by sonography and CT. Radiology 1985;157(1):63-8.

[13] Teele RL, Hernanz-Schulman M, Sotrel A. Echogenic vasculature in the basal ganglia of neonates: a sonographic sign of vasculopathy. Radiology 1988;169(2):423-7.

[14] Ivanov I, Zlatareva D, Pacheva I, et al. Does lenticulostriate vasculopathy predipose to ischemic brain infarct? A case report. Journal of Clinical Ultrasound 2012;40(9):607-10.
[15] Lingappa L, Varma RD, Siddaiahgari S, et al. Mineralizing angiopathy with infantile basal ganglia stroke after minor trauma. Developmental Medicine \& Child Neurology 2014;56(1):78-84.

[16] Yang FH, Wang H, Zhang JM, et al. Clinical features and risk factors of cerebral infarction after mild head trauma under 18 months of age. Pediatric Neurology 2013;48(3):220-6.

[17] Yang ST, Lee WT, Hung KL, et al. Infantile basal ganglia stroke after mild head trauma. Neurology 2015;84(23):2381-2.

[18] Landi A, Marotta N, Mancarella C, et al. Basal ganglia stroke due to mild head trauma in pediatric ageclinical and therapeutic management: a case report and 10 year literature review. Italian Journal of Pediatrics 2011;37:2.

[19] Chabrier S, Sébire G, Fluss J. Transient cerebral arteriopathy, postvaricella arteriopathy, and focal cerebral arteriopathy or the unique susceptibility of the $\mathrm{m} 1$ segment in children with stroke. Stroke 2016;47(10):2439-41.

[20] Sébire G, Fullerton H, Riou E. Toward the definition of cerebral arteriopathies of childhood. Current Opinion in Pediatrics 2004;16(6):617-22.

[21] Mallick AA, O'Callaghan FJ. Risk factors and treatment outcomes of childhood stroke. Expert Review of Neurotherapeutics 2010;10(8):1331-46.

[22] Braun KP, Bulder MM, Chabrier S, et al. The course and outcome of unilateral intracranial arteriopathy in 79 children with ischaemic stroke. Brain 2009;132(Pt 2):544-57.

[23] Ghosh M, Ghosh K, Chatterjee A, et al. Correlation of intracranial atherosclerosis with carotid stenosis in ischemic stroke patients. Annals of Indian Academy of Neurology 2015;18(4):412-4.

[24] Suresh P, Devi CY, Kumar CR. Study of the incidence of infarction versus haemorrhage in acute stroke. Journal of Evolution of Medical and Dental Sciences: JEMDS 2015;4(33):5630-3.

[25] Kumar LT, Gore VN, Patil GC. The role of computed tomography in the evaluation of cerebrovascular accidents. Int J Res Med Sci 2016;4(10):4305-9. 